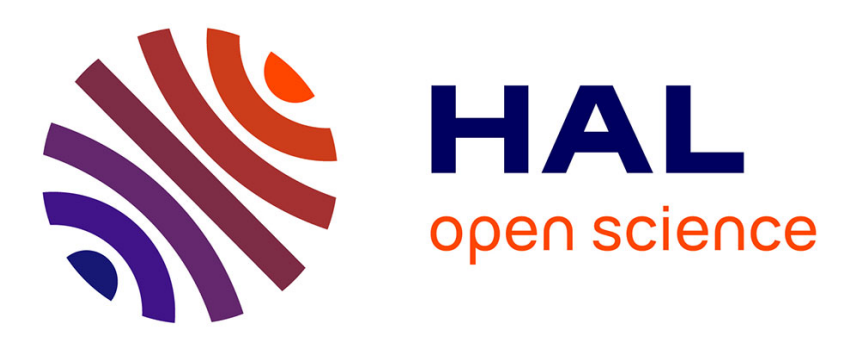

\title{
A model for problems' representation at various generic levels to assist inventive design
}

Sébastien Dubois, Philippe Lutz, François Rousselot, Gérard Vieux

\section{To cite this version:}

Sébastien Dubois, Philippe Lutz, François Rousselot, Gérard Vieux. A model for problems' representation at various generic levels to assist inventive design. International Journal of Computer Applications in Technology, 2007, 30 (1/2), pp.105-112. 10.1504/IJCAT.2007.015701 . hal-00794335

\section{HAL Id: hal-00794335 \\ https://hal.science/hal-00794335}

Submitted on 25 Feb 2013

HAL is a multi-disciplinary open access archive for the deposit and dissemination of scientific research documents, whether they are published or not. The documents may come from teaching and research institutions in France or abroad, or from public or private research centers.
L'archive ouverte pluridisciplinaire HAL, est destinée au dépôt et à la diffusion de documents scientifiques de niveau recherche, publiés ou non, émanant des établissements d'enseignement et de recherche français ou étrangers, des laboratoires publics ou privés. 


\title{
A model for problems' representation at various generic levels to assist inventive design
}

\author{
Sébastien Dubois ${ }^{1}$, Philippe Lutz $^{2}$, François \\ Rousselot $^{1}$, Gérard Vieux ${ }^{3}$ \\ ${ }^{1}$ Laboratoire de Génie de la Conception, INSA de Strasbourg, 24 bld de \\ la Victoire, 67000 STRASBOURG \\ ${ }^{2}$ Laboratoire d'Automatique de Besançon, UMR CNRS 6596, ENSMM, \\ UFC, 24 rue Alain Savary, 25000 Besançon \\ ${ }^{3}$ TRIXELL, Z.I. Centr'Alp, 38430 MOIRANS
}

\begin{abstract}
Inventive design requires specific competences. Designing means create new concepts out of acquired knowledge. To reach such a goal cognitive competences are required. TRIZ is a theory for inventive problem solving which is based on the abstraction of knowledge and of the description of the problems to solve, to be able to make analogies with any technical domain. This principle of abstraction is of great interest, but it also requires the ability to shift from a high level of abstraction to a more specific level. The building of the links between abstract models of problems and more specific ones is missing in TRIZ. This article proposes an UML model to make this link. Starting from a functional description of the systems, it enables the construction of abstract formulation of problems according to TRIZ frames. A case study illustrates the different description of the problems and the link between them.
\end{abstract}

Keywords: inventive design; TRIZ; UML model.

Reference to this paper should be made as follows: Dubois, S., Lutz, Ph., Rousselot, F., Vieux, G. (xxxx) 'A model for problems' representation at various generic levels to assist inventive design', Int. J. Computer Applications in Technology, Vol. X, No. Y, pp.000-000.

Biographical notes: Sébastien Dubois is Research Engineer in INSA Strasbourg graduate school of science and technology. He earned his $\mathrm{PhD}$ in Engineering Sciences from the University Louis Pasteur of Strasbourg. He teaches TRIZ knowledge to undergraduate and post-graduate students. He also participates to industrial inventive case studies. His research areas of interest include inventive design, problem resolution and innovation management.

\section{Introduction}

A lot of competences are required to design new technical systems [1-4]. Designing is being able to synthesize new concepts. Two kind of design can be distinguished implying different kind of competences: routine design and inventive design. Inventive design is particular in the sense that new ideas have to emerge. However these new ideas have to be built out 
of known things. This is a real paradox: engineers have to build new things in order to be able to design inventively but engineers do not have to build new things as they only have the existing knowledge. Regarding how inventive products are designed, one of the main principles is the application, in a new domain, of things known in another domain [5]. This principle has brought interesting results. How to be able to make bridges between different domains? It means how to be able to identify and recognize that one principle used in one domain could be useful and applicable in another domain. Moreover, engineers are specialized in the one domain in which that have got to solve problems, and looking for solutions from other domains are out of their competences. Even more, lot of psychological barriers disable human being to seek for solutions in a non mastered domain.

One theory for inventive problem solving in design of technical systems is TRIZ [6]. It is based on the building of analogies between different domains. To enable these analogies, TRIZ proposes to define the problem that has to be solved at a high level of abstraction. It means that TRIZ proposes both models to formulate the problems and principles of solution linked to these models. The basis of this theory is the analysis of millions of patents and the description of the problems solved by these patents in abstract shape. This analysis lead to the conclusion that, regarding problems and solutions at a high level of abstraction, there are not infinite numbers of generic problems as there is no infinite ways to solve these generic problems.

One of the main advantages of the use of TRIZ methods is to oblige engineers to think at different levels of abstraction. One of the main drawbacks of the use of TRIZ methods is to enable engineers to shift from their traditional models to the ones proposed by TRIZ methods.

The synthesis of a general model enabling a good understanding of the knowledge that has to be represented throughout the TRIZ problem formulation models and to explicit the links between these different models is a key stake. The analysis of the different software ([7-11]) to support TRIZ application (see [12]) shows that the software are complementary as each of it brings a partial support for the problem resolution steps. For example, none of the software consider the systemic description of the problem, which is though one of the core benefits of TRIZ models. 
A model for problems' representation at various generic levels

To overcome this drawback a model is proposed in this article to elicit the links between the different models of TRIZ for problem formulation and the functional description of systems.

\section{Specificities of inventive design}

Inventive design is specific in that it concerns open-ended, but ill-defined, problems [13]. The design problems are considered ill-defined since initially, designers only have an incomplete and imprecise mental representation of the design objectives. Designers' mental representations develop as the problem-solving process progresses [14]. This specificity in terms of design problems has been described as being based on an iterative dialectic between positioning the problem and finding a solution to the problem [15]. To summarize this approach, during the positioning of the problem, the designers refine the design objectives and its specifications and therefore refine their mental representation of the problem. During the problem-solving phase, designers work out solutions and assess these solutions in relation to a variety of criteria and constraints. Furthermore, the design problems are also defined in infinite space as no single correct solution can exist for a given problem, but rather a range of potential solutions is proposed. For this reason, inventive design problems are defined as open-ended problems.

These are the very characteristics of design problems that make them hard to solve and requiring high level competencies of analysis. Indeed, the problem is inadequately defined at the beginning and reformulation processes are therefore required to give a precise definition of the problem to be solved.

The reformulation processes mean that it is possible to move on from a description of the problem situation to a definition of the problem itself. The problem situation, which is a description of the objective to be reached without specifying the situation's mode of evolution can be distinguished from the problem, which defines a mode of evolution which will meet the objective; also the reason why it is impossible to achieve this evolution with known solutions will be explicit. This elicitation is the definition of the core of problem.

The formulation space is an open area - the formulation of the problem may differ for the same description of the problem situation. The path leading from the problem situation to the formulated problem depends on several factors. 


\section{S. Dubois et al.}

- The constraints integrated during the reformulation process - these constraints are specific to the problem situation.

- The strategic choices made by the designer - these choices are directed by the strategic policy at the time the problem is to be solved.

The knowledge of the designer solving the problem also guides the reformulation process. This knowledge is related to the problem and the field in which it occurs.

Gero, [16], defines conceptual design, the creative phase in the design process, as being characteristic of the fact that "all knowledge required to achieve the design is not known in theory, i.e. part of the design process consists in determining what is required." As specific knowledge are no sufficient to solve the problems, it is required to use more generic, more abstract, descriptions to be able to imagine new concepts and also to better understand and define the core of problems.

\section{Models of TRIZ}

TRIZ, a Russian acronym which means the Theory of Inventive Problem Solving [6], is a design theory centered on formulating and solving problems. In TRIZ several models for formulation of problems are proposed. Each of these models corresponds to a different level of abstraction. Some of the models are used to enable resolution by the use of shaped databases of generic solutions; some of the models are only intermediary steps in the problem formulation process. At the lowest level of abstraction, the more specific level, there is what is called, in TRIZ terminology, the technical contradiction. This level of problem formulation is an intermediary step in the resolution process, as this frame of problem formulation is dedicated to a better understanding of the problem without aiming at an inventive resolution of the problem. The technical contradiction is the expression of the opposition between two states of a system that seems impossible to satisfy at the same time. In TRIZ terminology, the two states are defined as being two parameters of the same system. Khomenko [17] has proposed a more precise definition of this level of definition of the problem, describing these parameters as evaluation parameters. Evaluation parameters are used to check if the problem is solved or not, but not used as parameters on which we can proceed to solve the problem. The simplest way proposed in TRIZ to formulate the problem through this shape is to explain why a known solution, a typical solution, can not be applied in the considered case. 
A model for problems' representation at various generic levels

A technical contradiction exists when a solution is known to reach the satisfaction of the evaluation parameter 1 , but the application of this solution disables the satisfaction of the evaluation parameter 2 .

For example a technical contradiction exists in belt-pinion systems when the rigidification of the belt enables a better transmission, but then disables the ability of the belt to turn around the pinion.

The next frame of problem definition, at a higher generic level, is the Vepole model, also known as Substance-Field Model. This frame is the representation of the interactions existing between the resources present in the problem. The resources are classified according to the fact they are a substance or a field. This model represents the nature of the interaction and the role (useful or harmful) it plays in the problem. The main objective of this model is to focus on the few elements directly implied in the problem. For this model of the problem, 76 rules to modify the interactions between the resources as required to solve the problem are proposed [18]. These generic rules are called the inventive standards.

A Vepole model is defined when some interactions between resources (which can be either substance or field) have to be modified.

For example, the interaction between the belt and the pinions is insufficient, as we need to rigidify the belt to provide a better transmission. The resources are two substances, the belt and at least one pinion, and also a mechanical field.

A last model is proposed in TRIZ and is called the physical contradiction. This model is the more abstract one. This model focuses the problem on one single element of the system, which is the core of problem. According to TRIZ a problem can always be formulated as a physical contradiction. Such a contradiction is defined as the requirement for one element to be in two contradictory states. The two benefits of such a formulation are:

- The focus on one single element, which is the core of problem

- The formulation that is totally not specific to a domain

A physical contradiction exists when one element of a system has to be in two contradictory states.

For example a physical contradiction exists in belt-pinion systems as the belt as to be both rigid to transmit energy efficiently and flexible to turn around the pinions.

Another interest of this level of formulation is that few principles exist to satisfy both contradictory states, in TRIZ a list of 11 principles is proposed. One of these principles is designing a system having globally 
S. Dubois et al.

one property but made of elements having the contradictory property. This principle is applied by the chain to solve the problem of the belt. The chain is globally flexible and made of rigid elements.

\section{Presentation of the UML model}

The built model [12] is the object-oriented representation of the TRIZ problem formulation frames. It does not include all the concepts of the theory; especially TRIZ proposes laws of evolution for technical systems, which are not available. The objectives are both to propose a clear description of TRIZ problem formulation frames that are proposed at different levels of abstraction, and to make the link between these frames and a functional description of systems.

The model is represented on figure 1, in UML (Unified Modeling Language), and is described below. The next description of the model is the description of the TRIZ frames to represent the real elements and the problems. In this model some peculiarities appear in regard of classical TRIZ frames. First of all, the technical contradiction model is not present; it has been replaced by a functional description of the systems. This proposal is based on the fact that the evaluation parameters of systems are a representation some functionalities of a specific system. Considering the example of the bicycle chain, ability to transfer energy was defined as an evaluation parameter, but it can also be described as a function. Thus the functions in the presented models are on the same level, and provide the same role of intermediary problem statement, as technical contradiction in classical TRIZ.

A resource is the basic element representing a real object characterized by its localization and described by a whole of parameters. A resource could be localized inside the system implied in the study, in an adjacent system or, more globally, in the environment. A resource could be a field, a geographical zone (a space), a temporal period (a time) or a material resource. The nature of the field could be magnetic, mechanic, electric, chemical or thermal. A material resource could be a system or a substance in regard of its decomposability. A system is composed of, at least, four material resources, enabling its functionality. These four main elements assume the roles of motor, transmission, tool and control to provide the function.

A function is the modification of the value of a parameter of a resource. The realization of the function modifies the parameter from an initial 
A model for problems' representation at various generic levels

value into a final value. The function operates during an operational time and within an operational zone. The importance of the function could be principal, if it is the function for which the system has been designed, or technical, if it is a sub-function enabling the realization of the principal one. The type of the function could be useful or harmful. It is harmful if it is a non desired function to be eliminated, resulting of the realization of other useful functions. A function could participate to the realization of a super-function and could be decomposed into sub-functions.

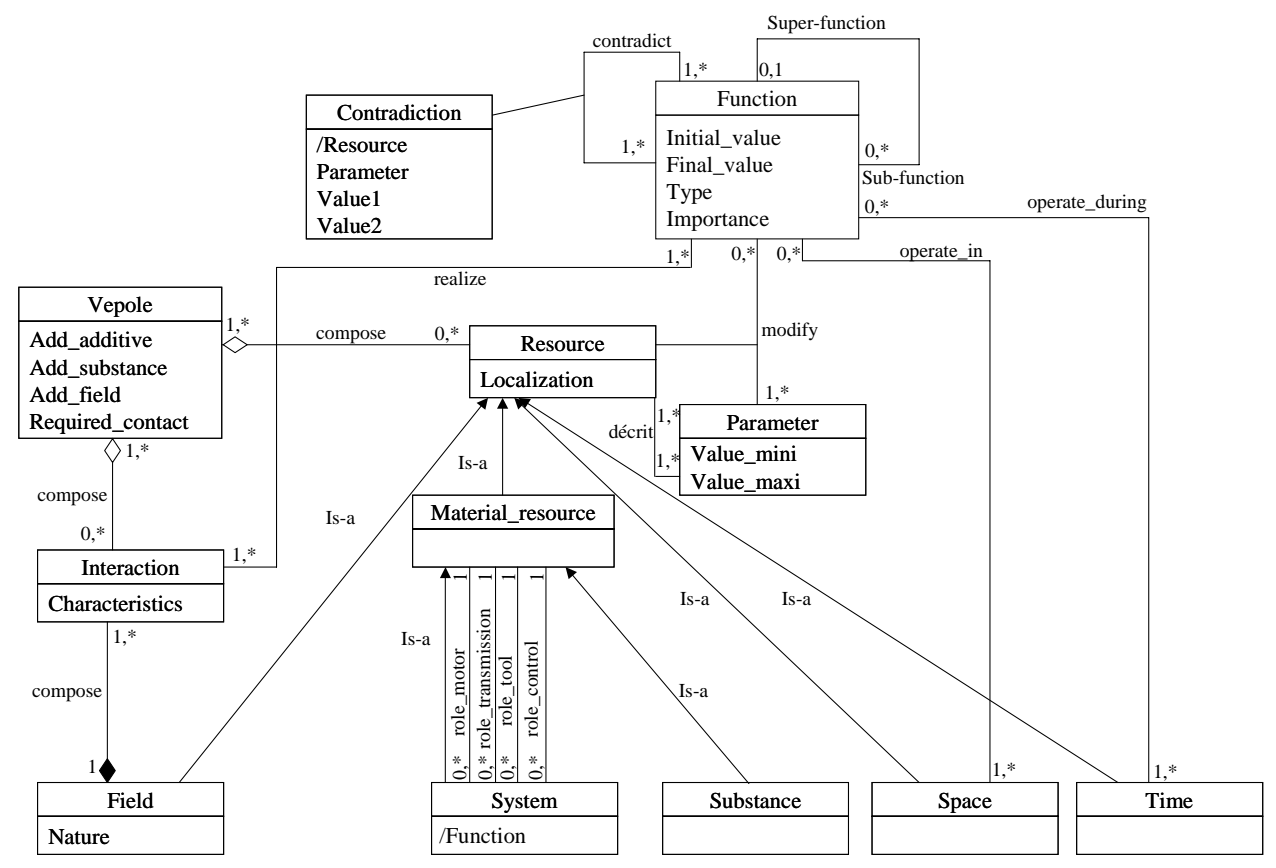

Figure 1. UML model of TRIZ frames

A contradiction arises if one or several functions require that the parameter of a resource has one value, when another, or several others, functions require this parameter to have another value.

An interaction is the representation of the action of a material resource on another material resource. This action is produced by a field. The interaction could be satisfying, excessive, insufficient or harmful. It is harmful if it is a non desired interaction, which is to eliminate (for example the Joule effect of a component inside an electric circuit). If the interaction is useful, it could be excessive, if it is realized more than necessary and then occurs disturbances (the sun radiations on the skin in summer, for example). A useful interaction could also be insufficient if its 


\section{S. Dubois et al.}

action has to be increased (the oxygen inflow in high altitude, for example). At last, if the interaction is to be kept as is, it is a satisfying one.

A Vepole model is made of interactions and of resources. This model is characterized by the additivity constrains and by the contact necessity. The possibilities to add a field, an additive or a substance are thus defined, as the possibility to break the contact between two substances of the Vepole model.

\section{Illustration}

TRIXELL develops and produces a complete family of X-ray flat panel digital detectors for the entire radiological imaging industry. TRIXELL has developed a real competitive strategy based on cost reduction. To reach this objective they are producing flat scanners made of assembled TFT plates. Using smaller TFT plates enable dramatic cost reductions, but imply developing algorithms to calibrate the scanners. To pursue their cot reduction strategy, TRIXELL was looking for a new principle of flat scanner production keeping their today's quality and reliability but requiring less calibration. One of the main difficulties for TRIXELL is to be able to imagine new solutions after ten years of development.

To solve their problems TRIXELL asked for TRIZ experts to help them in the description of their problematic situation and in the proposal of new concepts. As inventive concepts have been defined for which patents are in validation process, the results of this study won't be presented in this article. But the main objective of the article is to present the link between the different levels of problem formulation.

\subsection{General description of the problematic situation}

The studied system is a flat numeric scanner (cf. figure 2) used to scan human bodies to detect irregularities of $140 \mu \mathrm{m}$. It is made of several layers and at least 2 butted TFT plates. 
A model for problems' representation at various generic levels

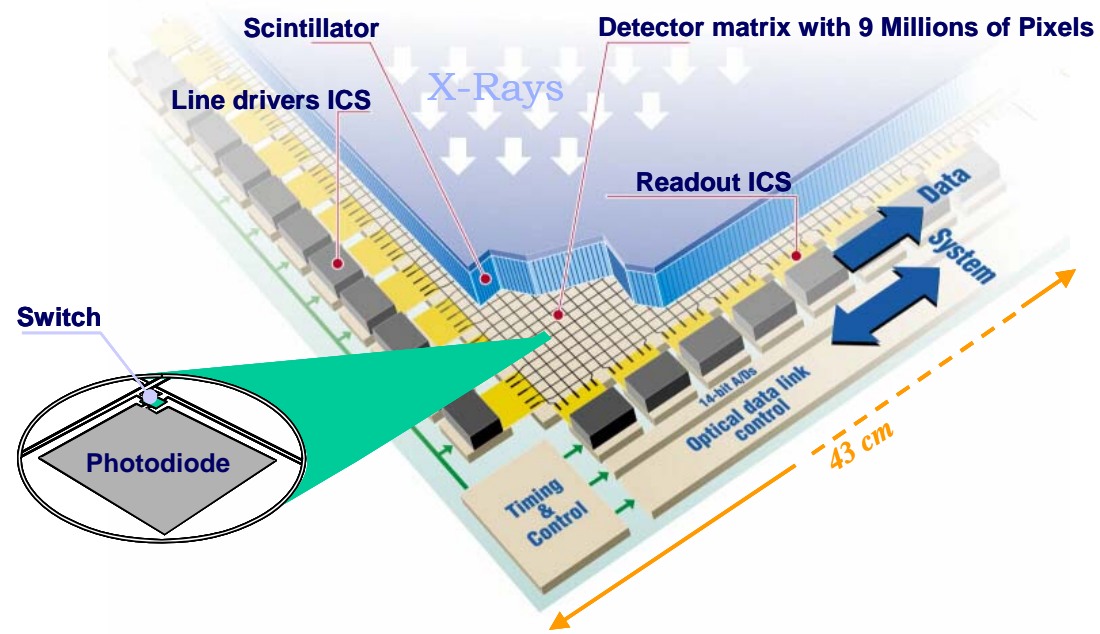

Figure 2. Main components of a flat numeric scanner

The main working principle is the transformation of the X-ray flow into an electric signal, and it is provided by a scintillator made of CsI which transforms the $\mathrm{x}$-ray flow into a visible light flow and of the TFT plates which transform the visible light signal into an electric signal, as illustrated on figure 3 .

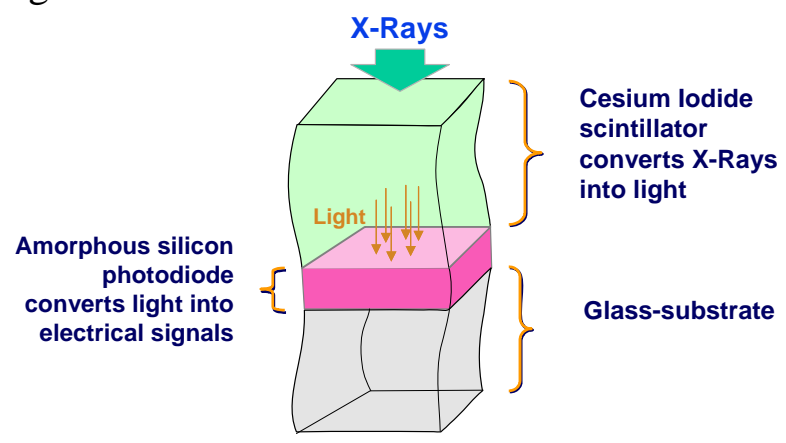

Figure 3. Main working principle of the flat numeric scanner

The use of several TFT plates enables the reduction of costs, as small TFT plates are less expensive to produce but in the same time it generates non homogeneous artefacts on the image, from one scanner to another. Thus specific algorithms have to be defined to calibrate each scanner.

The case study aim at designing a new flat numeric scanner made of several TFT plates, but taking off the need of calibration algorithms. 
S. Dubois et al.

5.2 Global description of the problem through the UML model

On figure 4 is presented a part of the modeled problem, which includes the definition of the main elements of the scanner and some of the underlined functions and problems identified throughout the analysis of the problematic situation.

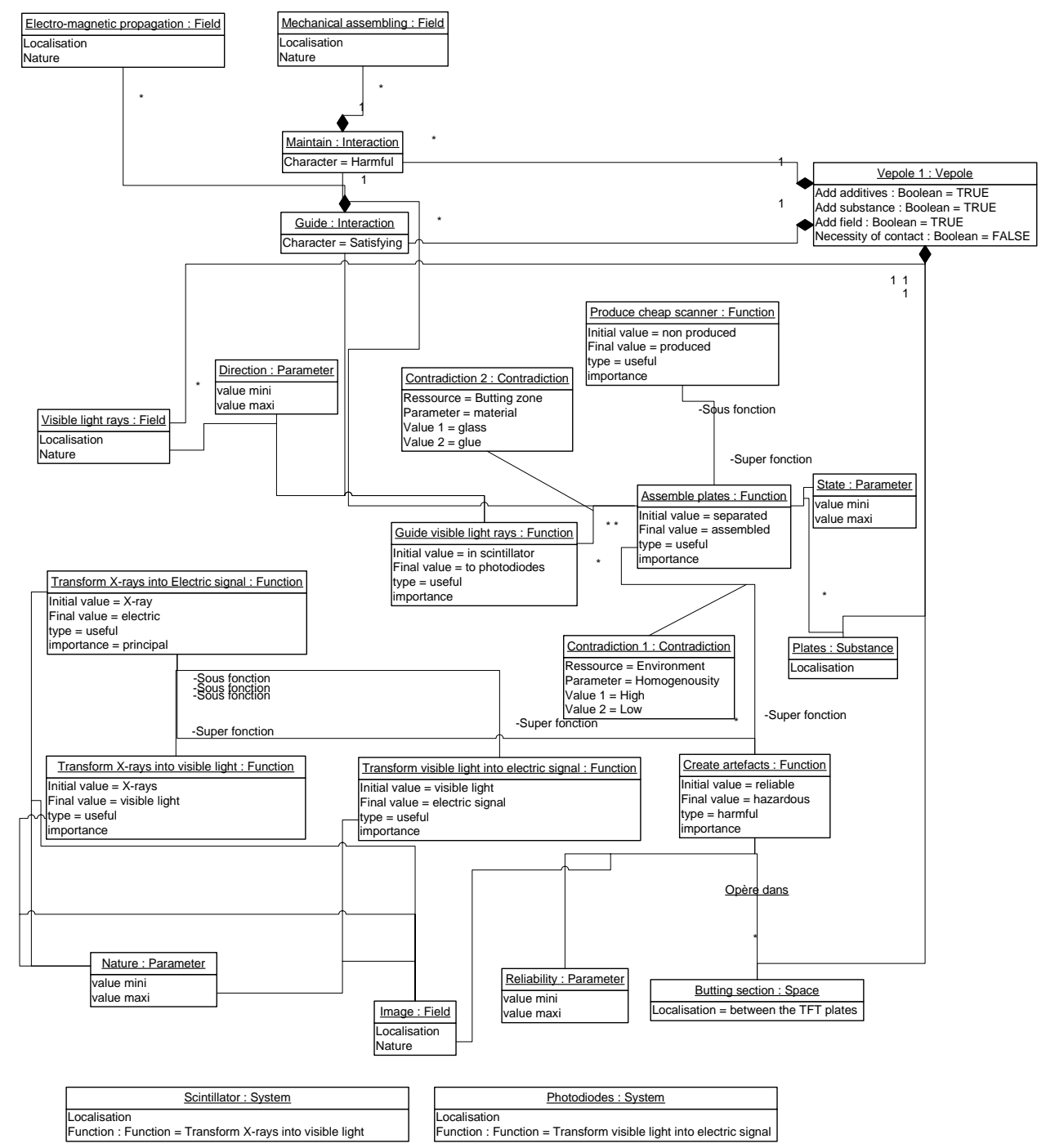

Figure 4. Global overview of the elements of the scanner

The building of the model has been guided by the use of two methods of TRIZ: 
A model for problems' representation at various generic levels

- The multi-screen scheme, which is based on a systemic analysis of problem, describing the implication of the problems on different systemic levels and according to its evolution in time.

- ARIZ, which is a method guiding the formulation of physical contradiction and the identification of the preferable resources to solve it.

\subsection{Description of the problem at different generic levels}

After an initial phase of general description of the system, of its components and of the objectives of the problem resolution, the first step in the process of problem clarification is the description of the specific required functions on which the problem focus.

A list of 6 functions has been listed as being main functions of the problem:

- produce cheap scanners

- transform X-rays into visible light rays

- transform visible light into electric signal

- assemble plates

- guide visible light rays

- create artefacts

A first peculiarity of this description is shown with the last listed function, as it is not a desirable function, but in reality it is performed and it has to be overcome. This function is identified as a harmful one.

For example, the functions "guide visible light rays" and "create artefacts" are described as shown on figure 5. 
S. Dubois et al.

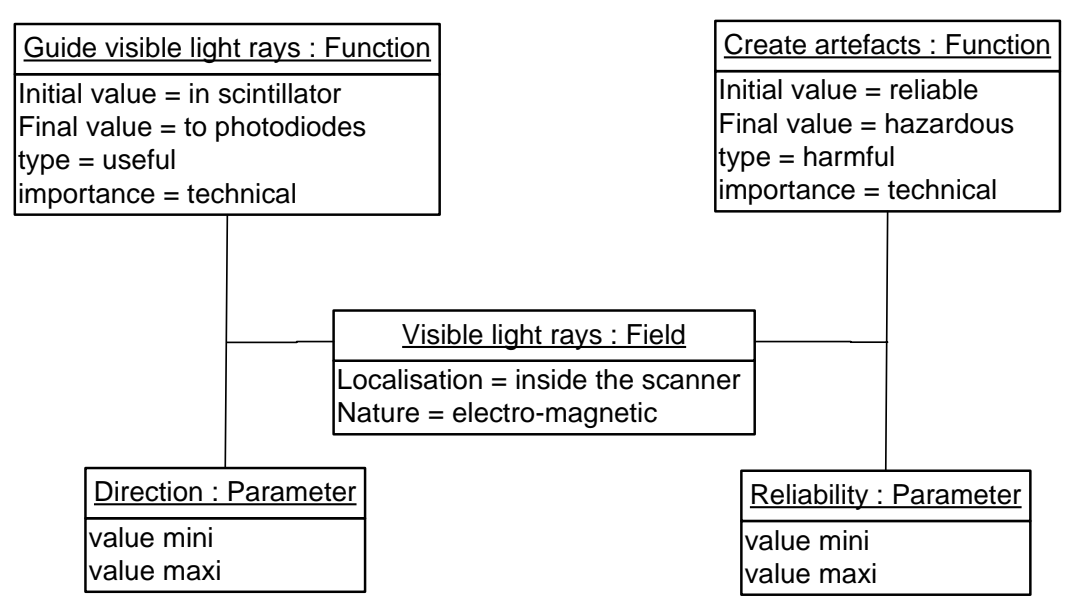

Figure 5. Functional level of problem formulation

A Vepole model could then be built and enables a clarification of the problem (cf. figure 6). In fact, the reason why artefacts appear is the necessity to assemble the plates. Each step of the problem formulation brings better understanding of the reasons why problem appears, and thus enables to define potential direction of resolution and elimination of the non relevant ones. 
A model for problems' representation at various generic levels

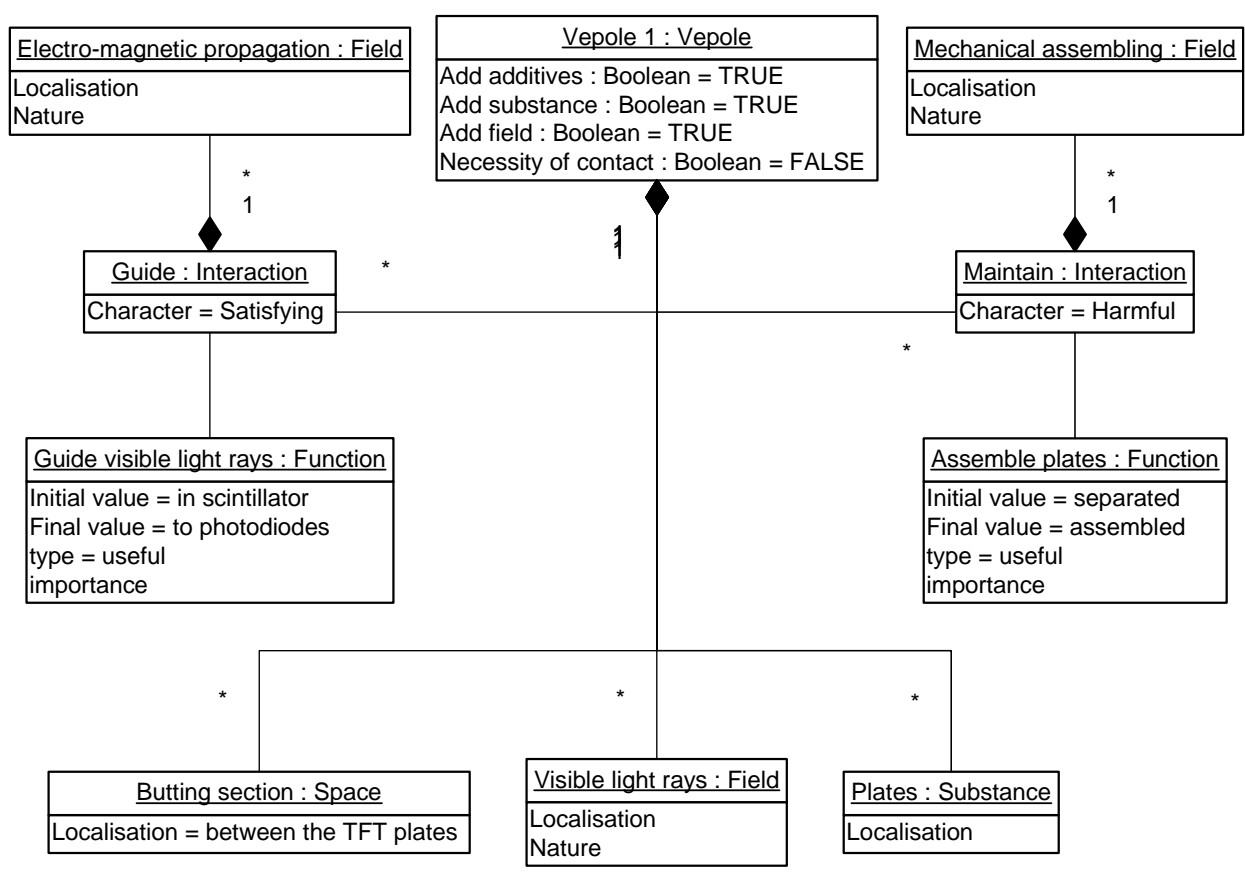

Figure 6. Vepole model of problem formulation

The last step in the problem formulation process is the formulation of the physical contradiction. This formulation point out that the core of problem is the homogeneity of the space around the scintillator and the TFT plates, which is called here the environment, as illustrated on figure 7.

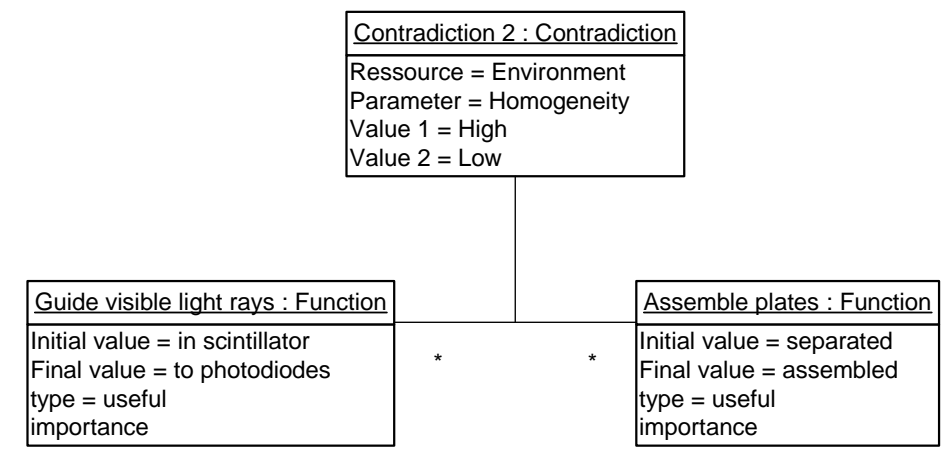

Figure 7. Contradiction model of problem formulation

The formulations of the previous Vepole model and of the physical contradiction enable a clarification of the role of each element of the system, and thus lead engineers from TRIXELL to a new understanding of 


\section{S. Dubois et al.}

the problem. Based on this understanding they were able to propose new concepts of solution.

As previously said these concepts won't be presented in the article but the objective was to explicit the different levels of problem formulation and the way it is used to shift from one level to another one. The three models of problem presented in figure 5 to 7 are all linked to the function "guide visible light rays".

\section{Conclusion}

The proposed model enables a clarification of the models of TRIZ for problem formulation. It also enables an easier transition from one model to another one. This second point is the most important one as it is from high importance to e able to transit from abstract to specific according engineers are looking for multi-domain analogies or applying generated inventive concepts to their specific situation. Moreover, this elicitation of the links between the models aims at facilitating the se of TRIZ models starting from a functional description of the systems.

As extension of this work, we are now studying how to link this model with a database in order to be able to build case-based reasoning for inventive problem solving, storing cases at different levels of abstraction. Also another direction of application is making the link between contradictions for problems of high complexity, and looking for rules to establish and manage such networks of contradictions.

\section{References}

1. Visser, W., Dynamic Aspects of Design Cognition: Elements for a Cognitive Model of Design. 2004, Institut National de Recherche en Informatique et en Automatique: Rocquencourt, France. p. 116.

2. Adams, R.S., J. Turns, and C.J. Atman, Educating effective engineering designers: the role of reflective practice. Design Studies, 2003. 24(3): p. 275-294.

3. Dörner, D., Approaching design thniking research. Design Studies, 1999(20): p. 407-415.

4. Greeno, J.G., Natures of problem solving abilities, in Handbook of learning and cognitive processes, W.K.E. (Ed.), Editor. 1978, Erlbaum: Hillsdale, NJ. p. 239-269.

5. Bonnardel, N. and E. Marmèche, Creative Design Activities: the Evocation and its Evolution with Regard to Expertise, in Computational and Cognitive Models of Creative Design V, J.S.G.a.M.L.M. (eds), Editor. 2001, Key Centre of Design and Cognition, University of Sydney, Australia. p. 189-204.

6. Altshuller, G.S., Creativity as an Exact Science. 1988, New York: Gordon and Breach.

7. Ideation, I.C., Innovation WorkBench. 2000. 
A model for problems' representation at various generic levels

8. Ideation, I.C., Improver. 2000.

9. Ideation, R.G., Knowledge Wizard. 2000.

10. Invention Machine, C., TechOptimizer Professional Edition. 1999: Boston.

11. TriSolver, C., TriSolver. 2002.

12. Dubois, S., Contribution à la formulation des problèmes en conception de systèmes techniques. Etude basée sur le TRIZ, in Sciences Pour l'Ingénieur. 2004, Université Louis Pasteur: Strasbourg, France.

13. Bonnardel, N., Towards understanding and supporting creativity in design: analogies in a constrained cognitive environment. Knowledge-Based Systems, 2000. 13: p. 505-513.

14. Simon, H.A., The structure of ill-structured problems. Artificial Intelligence, 1973. 4: p. 181201.

15. Simon, H.A. Problem Forming, Problem Finding, and Problem Solving. in $1^{\text {st }}$ International Congress on Planning and Design Theory. 1987. Boston, USA.

16. Gero, J.S., Conceptual designing as a sequence of situated acts, in Artificial Intelligence in Structural Engineering, I.S. (ed.), Editor. 1998, Springer: Berlin. p. 165-177.

17. Khomenko, N., Materials for Seminars: OTSM-TRIZ: Main Technologies of Problem Solving. 2001, "Jonathan Livingston" Project.

18. Altshuller, G.S., System of Standard Solutions for Inventive Problems. 1987, OTSM-TRIZ Technologies Center: Minsk, Belarus. 